\title{
Pulsatile ex vivo perfusion of human saphenous vein grafts under controlled pressure conditions increases MMP-2 expression
}

Sara Dummler ${ }^{1 * \dagger}$, Stefan Eichhorn ${ }^{1 * \dagger}$, Christian Tesche ${ }^{1}$, Ulrich Schreiber ${ }^{1}$, Bernhard Voss $^{1}$, Marcus-André Deutsch ${ }^{1}$, Hans Hauner ${ }^{2}$, Harald Lahm¹, Rüdiger Lange ${ }^{1}$ and Markus Krane ${ }^{1}$

* Correspondence: dummler@dhm. mhn.de; stefan.eichhorn@dhm. mhn.de

'German Heart Center Munich at the Technische Universität München, Department of

Cardiovascular Surgery, Lazarettstrasse 36, D-80636 Munich, Germany

Full list of author information is available at the end of the article

\begin{abstract}
Background: The use of human saphenous vein grafts (HSVGs) as a bypass conduit is a standard procedure in the treatment of coronary artery disease while their early occlusion remains a major problem.
\end{abstract}

Methods: We have developed an ex vivo perfusion system, which uses standardized and strictly controlled hemodynamic parameters for the pulsatile and non-static perfusion of HSVGs to guarantee a reliable analysis of molecular parameters under different pressure conditions. Cell viability of HSVGs $(n=12)$ was determined by the metabolic conversion of 3-(4,5-dimethylthiazol-2-yl)-2,5-diphenyl-tetrazolium bromide (MTT) into a purple formazan dye.

Results: Under physiological flow rates $(10 \mathrm{mmHg}$ ) HSVGs remained viable for two weeks. Their exposure to arterial conditions $(100 \mathrm{mmHg})$ was possible for one week without important reduction in viability. Baseline expression of matrix metalloproteinase-2 (MMP-2) after venous perfusion (2.2 $\pm 0.5, \mathrm{n}=5$ ) was strongly up-regulated after exposure to arterial conditions for three days (19.8 \pm 4.3 ) or five days $(23.9 \pm 6.1, p<0.05)$. Zymographic analyses confirmed this increase on the protein level. Our results suggest that expression and activity of MMP-2 are strongly increased after exposure of HSVGs to arterial hemodynamic conditions compared to physiological conditions.

Conclusion: Therefore, our system might be helpful to more precisely understand the molecular mechanisms leading to an early failure of HSVGs.

Keywords: Atherosclerosis, Bypass-Surgery, MMP, Perfusion, Pulsatile flow, vein graft

\section{Background}

Coronary artery bypass grafting (CABG) using venous grafts is a standard procedure in the treatment of advanced coronary artery disease. However, vein graft occlusion implanted in an arterial pressure environment is still a major problem [1]. Approximately 15 to $20 \%$ of human saphenous vein grafts (HSVGs) occlude within one month and $25 \%$ within the first year. Ten years after CABG about $50 \%$ of the HSVGs are occluded and $25 \%$ have been severely stenosed [2-6]. Early changes in vein grafts include endothelial disruption leaving the graft vulnerable to thrombotic incidents and smooth muscle cell (SMC) migration and proliferation from the media into the intima

(C) 2011 Dummler et al; licensee BioMed Central Ltd. This is an Open Access article distributed under the terms of the Creative Commons Attribution License (http://creativecommons.org/licenses/by/2.0), which permits unrestricted use, distribution, and reproduction in any medium, provided the original work is properly cited. 
within the first week after grafting $[1,7]$. The vein graft intimal thickening and remodeling occurs as an adaptation to increased wall stress and arterial flow with up to $15 \%$ of graft stenosis during the first year [8]. Under physiological conditions human saphenous veins are exposed to low pressure conditions $(\sim 5-10 \mathrm{mmHg})$, a nonpulsatile flow and a shear stress of 1-6 dyne $/ \mathrm{cm}^{2}$ [9]. After grafting and implantation into the coronary artery system the graft must support higher pressure conditions ( 60-140 $\mathrm{mmHg}$ ), a pulsatile flow and a shear stress range of 10-70 dyne $/ \mathrm{cm}^{2}$ during the cardiac cycle $[9,10]$. Beyond the first year after bypass surgery the development of graft atheroma and accordingly atherosclerotic vein graft stenosis is the dominant process underlying the failure of HSVGs [1,11]. Formation and evolution of atherosclerotic plaques are associated with variations in matrix metalloproteinase (MMP) expression. The gelatinases play a central role in matrix degeneration and SMC migration, a process which substantially contributes to vein graft failure. The involvement of different MMPs in vascular remodeling has been shown [12-14] whereas little is known about the specific role of gelatinases in HSVGs. While MMP-2 is either absent or only present at low levels in normal veins, its expression becomes elevated after graft implantation which may be a response to injuries during graft preparation or the exposure to the arterial environment [8]. It is generally accepted that the arterial mechanical environment plays a role in vein graft failure, yet the specific mechanical conditions and biological mechanisms have not been completely understood. Vessels cultured under static conditions have been widely used to study effects of pre-existing intimal hyperplasia (IH) [15]. Berceli et al. used a rabbit model to analyze intimal changes and MMP gene and protein expression after bilateral common carotid interposition vein grafting with defined regions of different wall shear [16]. The group of Patterson has used HSVGs in organ culture under static conditions or perfusion for seven days with the restriction of shear force calculation and the differentiation just between low-flow and high-flow conditions [17]. Compared to the animal model of Berceli et al. the $e x$ vivo perfusion system of Patterson et al. has a nonpulsatile hemodynamic environment, no blood-surface interaction and potential problems with delivery of nutrition or gas. Gusic and colleagues investigated the role of the mechanical environment in vein remodeling in a higher developed ex vivo perfusion system with a main focus on medial and intimal growth in the perfused veins. They ran their perfusions system with five different ex vivo hemodynamic environments and showed that pressure and shear stress act independently to regulate vein remodeling [7]. Yet, their study had the limitation of unstable pressure profiles during the course of the experiment. In the present study we have developed an ex vivo perfusion system which can be used to perfuse HSVGs with tightly controlled, steady and standardized perfusion profiles. We have defined the viability time course of perfused HSVGs exposed to arterial and venous perfusion profiles. In addition, we provide evidence that our system is suitable to detect alterations of molecular markers such as MMP-2 as a consequence of preparative injury or increased arterial perfusion pressure.

\section{Methods}

\section{Tissue Preparation}

Nonvaricose HSVGs were obtained from 35 patients (mean age 71.4 \pm 7.7 years; nine females, 26 males) undergoing CABG surgery in the German Heart Center Munich. 
The endoscopically harvested vein grafts were kept in autologous blood at room temperature until implant. One part of the graft was immediately stored in Ringer solution on ice and transferred from the operating room to the laboratory. One small piece was directly snap-frozen in liquid nitrogen and stored at $-80^{\circ} \mathrm{C}$ until further use as unperfused control tissue. This piece served as a reference to determine relative gene expression. The other part of the vein was mounted into the perfusion device as described. The procedure was acknowledged by the local ethical committee (Ethikkommission der TU München, Project no. 1588/06).

\section{Ex vivo perfusion system}

The circuit of the perfusion system is driven by a roller pump ISMATEC S2 (Wertheim, Germany) producing a pulsatile and non-static flow. All silicon tubings and the vessel chamber are sterilized prior to use. The vessel mounting procedure is carried out under a biological safety cabinet (NuAire, Plymouth, MN). Constant pressure conditions are maintained using a syringe pump (MC Medizintechnik $\mathrm{GmbH}$, Alzenau, Germany). The entire system is placed into a styrofoam-isolated chamber to maintain a constant temperature of $37^{\circ} \mathrm{C}$. Disposable pressure sensors (DPT-9300, Codan Critical Care, Forstinning, Germany) are placed on both sides of the vessel chamber to permanently monitor and facilitate the control of pressure conditions of the circuit. All functions and settings are controlled by a PC with a program written in java. Pressure is controlled by a PID-algorithm, data are logged continuously.

\section{Perfusion of human saphenous vein grafts}

HSVGs were fixed in the perfusion device by suture ligation (Ethibond Vicryl 3-0, Ethicon GmbH, Norderstedt, Germany) and adjusted to a length matching the in vivo conditions. Total time from operating room to perfusion was less than one hour. The perfusion medium was DMEM/Ham's F-12 (PAA, Marburg, Germany) supplemented with 10\% FCS, $2 \mathrm{mM}$ glutamine and antibiotics $(100 \mathrm{U} / \mathrm{ml}$ penicillin and $100 \mu \mathrm{g} / \mathrm{ml}$ streptomycin). Veins were perfused with venous conditions (flow: $5 \mathrm{ml} / \mathrm{min}, 10 \mathrm{mmHg}$, $\mathrm{n}=12$ ) or with arterial conditions (flow: $50 \mathrm{ml} / \mathrm{min}, 100 \mathrm{mmHg}, \mathrm{n}=12$ ) for various time periods. At the end of each experiment vein ends were discarded. The other part of the vein was snap-frozen in liquid nitrogen and stored at $-80^{\circ} \mathrm{C}$ until further use. In long-term experiments the medium was replaced every two days. The $\mathrm{pH}$ of the medium remained stable within this period.

\section{Determination of viability of vein grafts and histology}

To verify tissue viability, a staining with MTT (Sigma, Munich, Germany) was performed. In the presence of metabolically active viable cells the yellow MTT is converted into a water-insoluble purple formazan product due to reduction by mitochondrial dehydrogenases and other cellular enzymes [18,19]. MTT was stored as a stock solution $\left(5 \mathrm{mg} / \mathrm{ml}\right.$ in PBS) at $-20^{\circ} \mathrm{C}$. Short segments of veins $(\mathrm{n}=12)$ were incubated in MTT diluted in serum-free medium to $0.5 \mathrm{mg} / \mathrm{ml}$ for one hour at $37^{\circ} \mathrm{C}$. To analyze potential degenerative changes in perfused vessels, sections of formalin fixed and paraffin-embedded samples were analyzed after a conventional hematoxylin/ eosin staining. 


\section{Quantitative RT-PCR analysis}

Frozen tissue pieces were minced using a Precellys 24 lysis and homogenization system (Peqlab, Erlangen, Germany) and total RNA was extracted using Trifast according to the manufacturer's recommendation (Peqlab). All RNA preparations were digested with DNase I prior to cDNA synthesis using Omniscript RT kit (Qiagen, Hilden, Germany). One $\mu$ l of cDNA was amplified on a LightCycler 1.5 thermo cycler (Roche Diagnostics, Mannheim, Germany) using the QuantiTect SYBR Green Kit (Qiagen) and BSA $(0.5 \mathrm{mg} / \mathrm{ml})$ in a final volume of $20 \mu \mathrm{l}$. All primers were used in a final concentration of $0.5 \mu \mathrm{M}$. The following primers were used: $\beta$-actin forward 5' CCA ACC GCG AGA AGA TGA 3', $\beta$-actin reverse 5' CCA GAG GCG TAC AGG GAT AG 3', MMP-2 forward 5' TGC TGG AGA CAA ATT CTG GA 3', MMP-2 reverse 5' GAT GGC ATT CCA GGC ATC 3'. They amplify fragments of 96 and 90 bp, respectively. After an initial activation of $\operatorname{Taq}$ polymerase for $15 \mathrm{~min}$ at $95^{\circ} \mathrm{C}$ specific products were amplified during 40 cycles using the following conditions: $15 \mathrm{sec}$ at $94^{\circ} \mathrm{C}$ (denaturation), $20 \mathrm{sec}$ at $60^{\circ} \mathrm{C}$ (annealing) and $20 \mathrm{sec}$ at $72^{\circ} \mathrm{C}$ (elongation). The relative expression levels of MMP-2 in individual samples were calculated in relation to the expression of the $\beta$-actin housekeeping gene. To compare independent samples the ratios of MMP-2/ $\beta$-actin were calculated.

\section{Zymography}

MMP-2 protein activities were evaluated by a standard gelatine zymography. Briefly, $100 \mathrm{mg}$ of frozen HSVG tissue were homogenized in ice cold zymogram buffer (150 $\mathrm{mM} \mathrm{NaCl}, 1 \mu \mathrm{m} \mathrm{ZnCl}$, $1.5 \mathrm{mM} \mathrm{NaN}_{3}, 20 \mathrm{mM} \mathrm{CaCl}_{2}, 0.01 \%$ Triton X-100, $10 \mathrm{mM}$ cacodylic acid, $\mathrm{pH} 5.0$ ). Samples were centrifuged at $4^{\circ} \mathrm{C}$ for $10 \mathrm{~min}$ at $20.000 \times \mathrm{g}$. The supernatant containing proteins was removed and stored at $-80^{\circ} \mathrm{C}$ until further use. Ten $\mu \mathrm{g}$ of extracted protein were mixed with zymogram loading buffer (62.5 mM Tris/HCl, pH 6.8, 25\% glycerol, 4\% SDS, 0.01\% bromophenol blue) and separated in 15\% SDS-PAGE gels containing $1 \mathrm{mg} / \mathrm{ml}$ type A gelatine from porcine skin (SIGMA-Aldrich, Taufkirchen, Germany). To renature proteins, gels were washed two times in $2.5 \%$ Triton $\mathrm{X}-100$ for $15 \mathrm{~min}$ at room temperature and subsequently incubated in developing buffer, $\mathrm{pH} 7.5(200 \mathrm{mM} \mathrm{NaCl}, 50 \mathrm{mM}$ Tris, $5 \mathrm{mM}$ $\mathrm{CaCl}_{2}, 0.02 \%$ Brij-35) overnight at $37^{\circ} \mathrm{C}$. Gels were stained with $0.5 \%$ Coomassie Blue $\mathrm{R} 250$ in $40 \%$ methanol/10\% acetic acid for $15 \mathrm{~min}$ and destained in $40 \%$ methanol/ $10 \%$ acetic acid until clear bands of lytic activity appeared. The reaction was stopped by transfer of gels in aqua bidest. Gelatinolytic activity was quantified using Image software (version $1.43 \mathrm{u}$, National Institute of Health). The pixel intensities of bands within each gel were normalized against the respective control of unperfused venous tissue.

\section{Statistical analysis}

For the analysis of gene expression levels and MMP-2 gelatinolytic activity the comparison was made using the unpaired Student's $t$-test. Differences in the vessel viability were calculated using the Mann-Whitney U-Test. Differences were considered to be significant at values of $p<0.05$. 


\section{Results}

\section{Establishment of the ex vivo perfusion system}

Twenty four veins from twenty three patients were used for the ex vivo perfusion experiments to establish and proof the reliability of the system. The veins were fixed on tapered conical metal adapters with circular striae to ensure a tight fit of the grafts throughout the whole experiment (Figure 1A). All components used in the vessel chamber are biocompatible (PEEK, $316 \mathrm{~L}$ ) thereby avoiding any potential interactions with the veins. The grafts were brought to their initial length using the adjustment device. Deaeration was performed by using two three way stop cocks. An overview showing the components of the perfusion system is given in Figure 1B. Under arterial pulsatile (Figure 1C) and non-static flow conditions three veins were cultured for one day, five veins for three days and four veins for five days. To establish the reliability of the system we perfused five HSVGs for one, three veins for three and four veins for five days with low pressure conditions $(10 \mathrm{mmHg}$, flow rate $5 \mathrm{ml} / \mathrm{min}$ ) which mimics the physiological venous pressure profile. Sensors on both side of the vessel chamber permanently surveyed the pressure inside the circuit (Figure 1B). In case of a pressure decrease a tiny volume of medium was injected into the circuit from an external medium reservoir mounted in a syringe pump. With this setup we were able to maintain the pressure constantly within a deviation of less than $2 \mathrm{mmHg}$ during the whole experiment. The perfusion conditions were controlled by a customized software package. By using a PID control algorithm to control the syringe pump a constant pressure could be secured throughout the whole experiment. Pressure data were logged every 10 seconds and were analyzed after every trial.

\section{Human saphenous veins support arterial perfusion conditions for one week}

Under venous conditions all tested veins contained viable cells throughout the vessel wall for up to 12 days indicated by a conversion of MTT into a purple formazan product (data not shown). Thereafter, the viability dropped (Figure 2A). We then analyzed to what extent the veins would support an elevated pressure which corresponds to the arterial situation $(100 \mathrm{mmHg}$, flow rate $50 \mathrm{ml} / \mathrm{min})$. After one and four days of arterial perfusion all veins were fully viable (Figure $2 \mathrm{~B}$ ) and showed an intensive purple staining. Even after seven days the cells clearly showed metabolic activity though to a reduced degree (Figure 2B). Beyond one week the veins did not support these elevated pressure conditions evidenced by the complete lack of MTT conversion (Figure 2B). Thus, we have successfully established a standardized system, which allows the perfusion of human veins with an arterial pressure profile for up to one week.

To further explore potential pathological changes in HSVGs upon perfusion, we investigated tissue sections from veins after perfusion with venous or arterial pressure profiles at different time points by a hematoxylin/eosin staining. As a reference, we used an unperfused section of the same vein. Exposure to venous pressure for three days did not change the histology and even after five days a minor thickening of the intimal layer was evident (Additional File 1, Figure S1). After arterial perfusion for one day also no major changes could be noticed. However, after three days the intimal layer started to visibly thicken and after five days extensive hyperproliferative areas were seen (Additional File 1, Figure S1). 


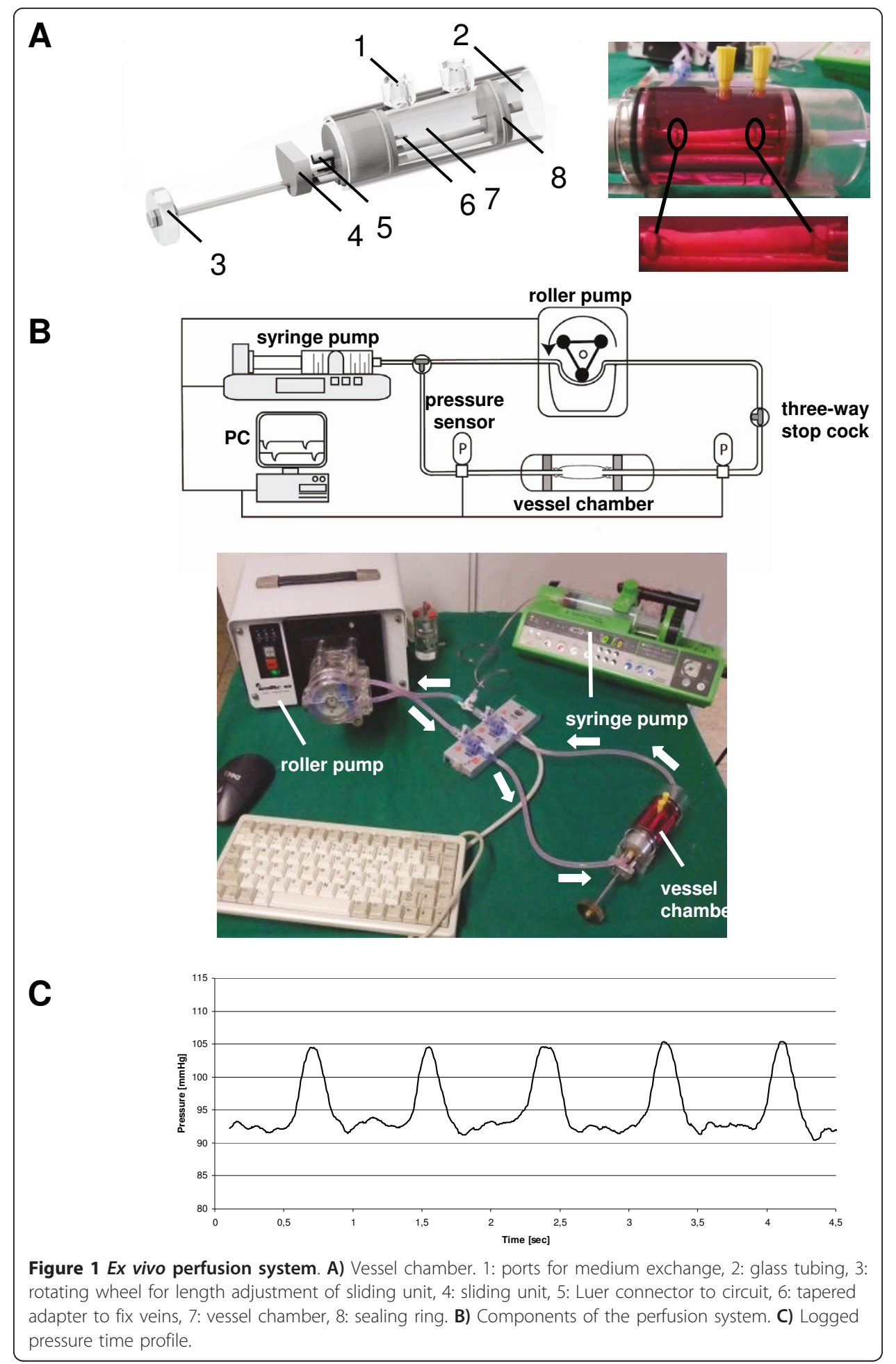

Arterial perfusion conditions up-regulate MMP-2 gene and protein expression

We next addressed the question whether the system is suitable to record alterations in gene expression as a consequence of exposure to different pressure profiles. To that end we analyzed MMP-2 as its expression is known to increase as a consequence of hypertension and vein graft preparative injury [20-22]. We first determined MMP-2 


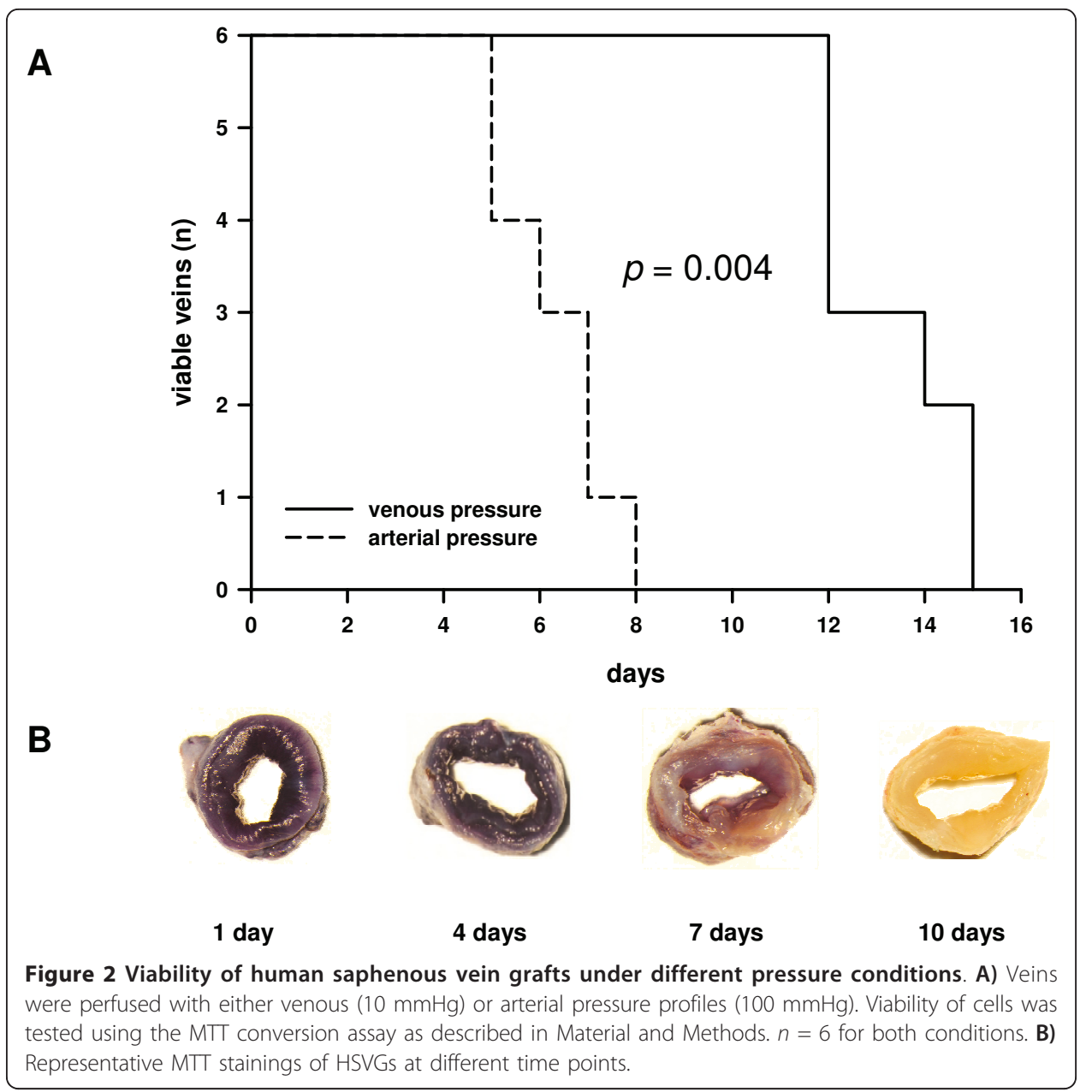

expression in human veins which were perfused with $10 \mathrm{mmHg}$ for one day which revealed a baseline ratio of MMP-2/ $\beta$-actin of $2.2 \pm 0.5(n=5)$ compared to unperfused control tissue (Figure 3). Extended perfusion of HSVGs for three days gave a similar result $(3.7 \pm 0.6, \mathrm{n}=3)$ and perfusion for five days under venous conditions showed a slightly increased gene expression of $5.0 \pm 1.0(\mathrm{n}=4)$ (Figure 3). No significant difference could be observed between venous perfusion of HSVGs for one or three days. Perfusion with $10 \mathrm{mmHg}$ revealed statistical significance between five days and one day $(\mathrm{p}<0.05)$ (data not shown), probably due to the elongated exposure in the ex vivo system. Perfusion of HSVGs with $100 \mathrm{mmHg}$ for one day yielded an MMP2 gene expression ratio which was similar to the reference $(2.4 \pm 0.4 ; \mathrm{n}=3$ ) (Figure 3). However, MMP-2 gene expression was significantly up-regulated when HSVGs were exposed to an arterial perfusion profile for three days $(19.8 \pm 4.3 ; \mathrm{n}=5)$. This value increased further when arterial conditions were extended to five days $(23.9 \pm 6.1 ; \mathrm{n}=$ 4) ( $<$ 0.05; Figure 3). Thus, the elevation of MMP-2 gene expression starts rapidly when HSVGs are exposed to arterial flow conditions and it is maintained at this high level for at least five days. We then determined whether this change in RNA expression was also reflected on the protein level in a zymographic analysis. Under venous pressure MMP-2 activity corresponding to a molecular weight of $72 \mathrm{kD}$ was detected, corresponding the activity of pro-MMP-2 (Figure 4A). Exposure to an arterial pressure 


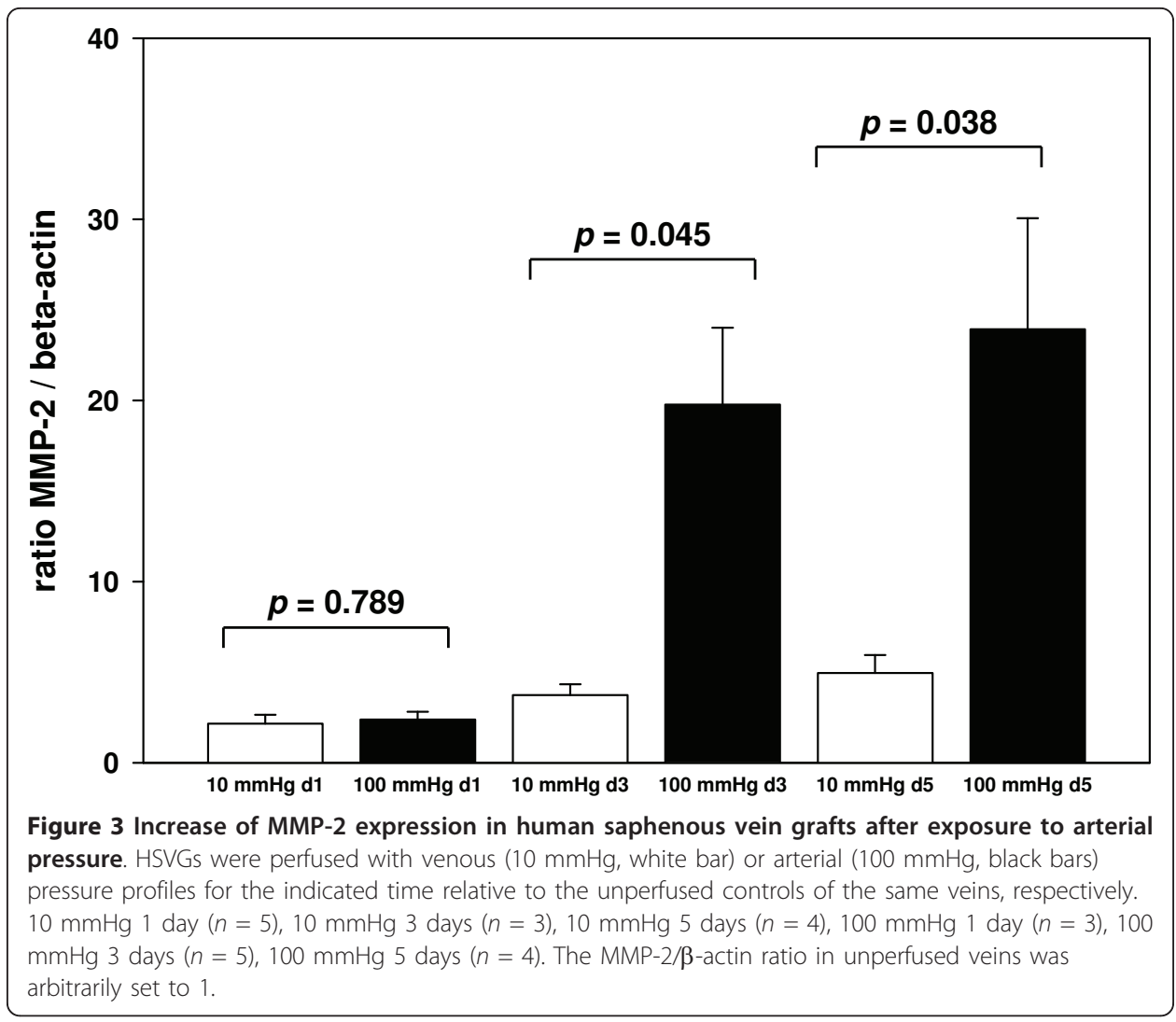

for one day yielded similar patterns (Figure 4A). However, when arterial pressure profiles were applied for three or five days gelatinolytic activities were strongly increased. In particular, the $63 \mathrm{kD}$ form of MMP-2 showed a heavily increased activity when compared to unperfused control tissues (Figure 4A). Quantification of the gelatinolytic activity confirmed our results of MMP-2 mRNA expression (Figure 4B). Gelatinase activity did not increase significantly between venous $(2.1 \pm 0.4, \mathrm{n}=5)$ and arterial $(3.4 \pm 1.4, \mathrm{n}=5)$ perfusion after one day. According to the results of mRNA expression extended perfusion with arterial pressure for three $(4.5 \pm 1.4, \mathrm{n}=5)$ or five days $(5.6 \pm 0.9, \mathrm{n}=5)$ revealed significantly elevated MMP-2 gelatinolytic activity compared to venous conditions $(2.5 \pm 0.6, \mathrm{n}=5$ and $2.7 \pm 0.4, \mathrm{n}=5$, respectively).

Thus, our novel ex vivo perfusion system proved its ability to monitor alterations in the expression of genes which are expected to increase their activity due to elevated pressure conditions on the RNA and protein level.

\section{Discussion}

A major problem with HSVGs remains their occlusion after a certain time. Transposition of a vein segment and exposure to the arterial hemodynamic environment leads to an acute increase in flow rates and intraluminal pressure and is thought to be a potential trigger for the pathological remodeling of HSVGs [22,23]. Gene expression profiling approaches revealed that many genes and multiple pathways are differentially regulated under these conditions [24-26]. In the present study, we have established an ex vivo perfusion system developed to mimic the arterialization of HSVGs. The culture system provides the ability to reproduce the initial events taking place when the grafted 


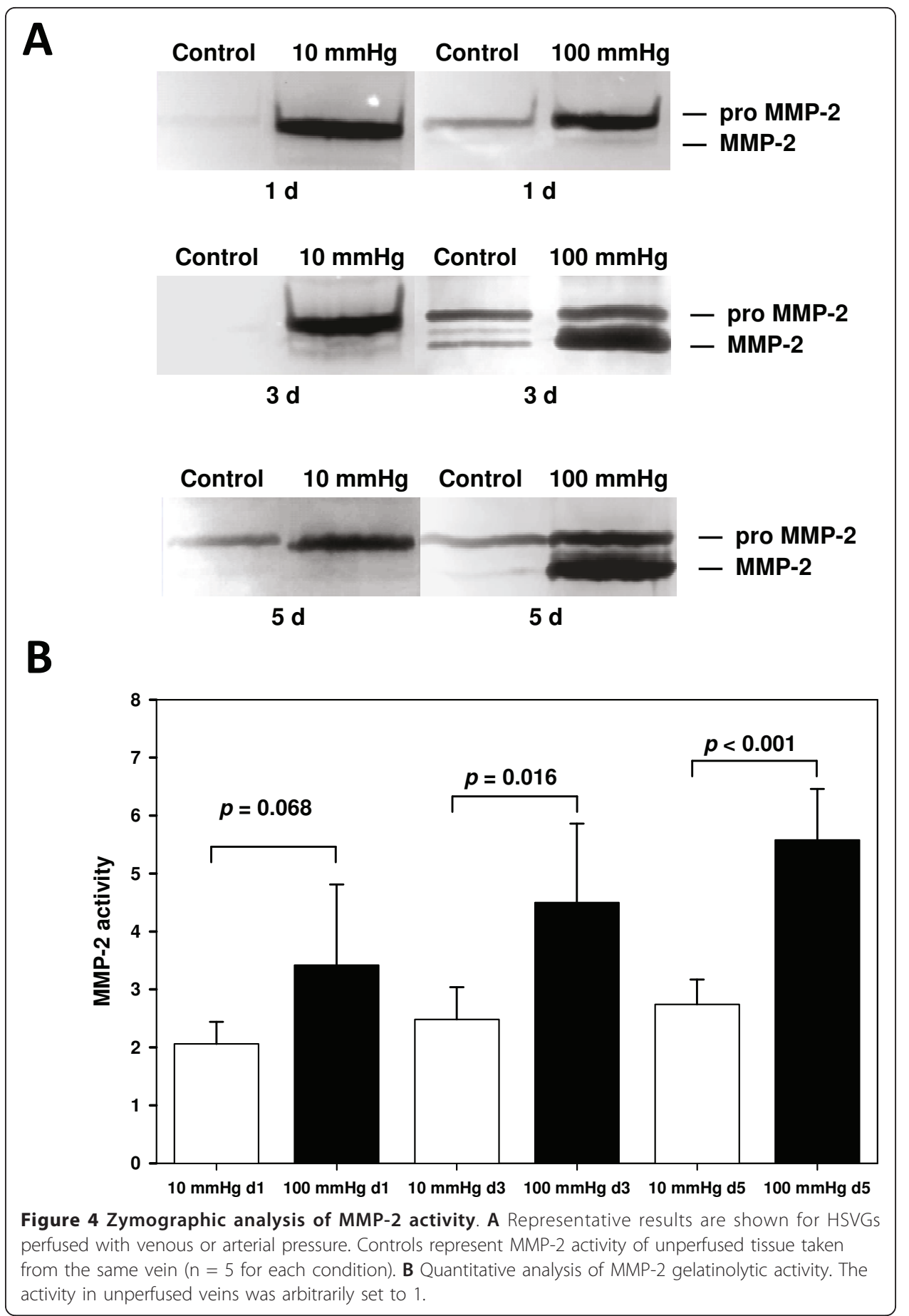

vein is exposed to arterial hemodynamic conditions. Therefore, our system may represent a valuable and reasonable approach to identify molecular mechanism underlying the early stages of bypass grafting. Several in vitro and in vivo studies have demonstrated changes in graft morphology, viability, cellular density or gene expression under arterial conditions $[7,16,22,27,28]$. Saucy et al. for instance used an ex vivo vein support system to perfuse HSVGs with arterial conditions regarding shear stress, flow rate and pressure during a period of 7 and 14 days. They found significant $\mathrm{IH}$ and a 
marked increase in plasminogen activator inhibitor 1 (PAI-1) expression in the human veins after 7 and 14 days of perfusion [29]. A mathematical model of early vein graft IH induced by shear stress and based on experimental data with bilateral rabbit carotid vein grafts describes the general behavior of the remodeling process [23]. The group of Porter et al. demonstrated that arterial shear stress inhibits the development of IH in cultured vein pieces [30]. Previous studies have shown that SMC proliferation and migration depend on the activity of matrix-degrading enzymes. In fact, MMP-2 is an enzyme which is directly involved in vascular remodeling [14] and rodent animal models confirm that MMP-2 levels are increased under hypertensive conditions [20,21].

Within three days of perfusion under arterial pressure conditions in our perfusion system the expression of MMP-2 increased more than nine-fold and reached an even higher value after five days, similar to the activation of PAI-1 [31]. Our data are further supported by other reports which shows an increased de novo synthesis of MMP-2 in HSVGs perfused with artertial conditions [22] or in animal models who underwent vein grafting $[8,16]$. Berceli et al used a rabbit model with bilateral common carotid interposition vein grafting. They could show that accelerated IH resulting from reduction in wall shear stress was associated with an increase in MMP-2, mainly in an active form [16]. Our zymographic analyses are in accordance with their results and those of Patterson et al. [17], as we found strongly increased gelatinolytic activities in veins after perfusion with arterial pressure profiles particularly of the active form of MMP-2. As we compared HSVGs under venous or arterial pressure conditions, the elevation of MMP-2 can be attributed strongly to the arterial pressure profile. Both, gene and protein expression were significantly increased after perfusion with an arterial hemodynamic profile compared to venous conditions although all HSVGs had the same mechanical injuries after harvesting and mounting in the ex vivo perfusion system. Thus, the results of our perfusion system perfectly reflect the in vivo situation suggesting that genes which are involved in vascular remodeling are activated by arterial pressure. Therefore, our system can be used to analyze molecular parameters involved in such events in detail under standardized, tightly controlled and reproducible conditions.

An important advantage of our system is the possibility to mount vessels of variable length and diameter. The sliding unit allows a very flexible adjustment to guarantee that the vessel maintains its natural length and tension throughout the experiment. Our main focus was to setup an experimental system, which is suitable to reliably analyze molecular parameters as a function of altered pressure and flow conditions. Therefore, the most important point was to control the pressure conditions very stringently and also to keep them very stable. In pilot experiments we experienced a continuously decreasing pressure in the circuit, despite any leakage. Knowing that pressure affects gene expression such a behavior would be fatal for a desired molecular readout. With regard to this a unique feature of our perfusion system is the regulation of the mean pressure in the circuit by a computer controlled syringe pump. Decreasing pressure due to diffusion processes through out the silicone tubing [32] or relaxation of the vessel can be compensated automatically. Long time trials can be performed due to this amendment enabling an objectively constant mean pressure. In addition, up to four grafts can be perfused simultaneously within one circuit. Using MTT conversion we were able to confirm that HSVGs, which were perfused with a low-pressure profile in 
our system, remained viable for up to two weeks. This is in good agreement with other reports which have estimated the integrity of the vessels by histological or immunohistochemical methods $[28,31,33]$. Switching the conditions to an arterial pressure profile leads to a visible reduction of the MTT staining beyond five days of perfusion. These findings are similar to those of Miyakawa et al. who detected diminished cell viability in vein segments after perfusion with arterial conditions for four days [28]. They confirmed their results by hematoxylin staining which also reveals a reduction of nuclear staining on day four [28]. Gusic et al. could even show a dramatic increase in cell death index in all layers of the graft after one week [7]. We have also performed experiments in which HSVGs were perfused with pathologically elevated pressure (200 $\mathrm{mmHg}$ ). However, under these conditions the grafts rapidly degenerated and after two days no MTT conversion was detected any more (data not shown). Our study, like others, is limited by the inability to perfuse the ex vivo system with autologous blood lacking blood cells, platelets, plasma, blood-surface interaction and the multitude of inflammatory and coagulation mediators playing an important role in the pathophysiology of IH development. However, because of technical reasons, we were not able to perfuse veins with blood by using a roller pump for perfusion to achieve a pulsatile flow. Platelets would be inevitably activated and blood cells destroyed during passage through the pump. Hemolysis could be avoided or highly reduced by using a centrifugal pump instead, which in turn produces a nonpulsatile flow. Inclusion of blood would provide exposure of the vein to a more physiological state, but may also confound the results with numerous other variables. Another limitation is the timerestricted viability of grafts in the ex vivo perfusion system which would not be prolonged by blood perfusion due to the accumulated metabolic waste products and inflammatory reactions. Despite these limitations, the findings of the current study highlight important potential in our understanding of the healing and adaptation of veins transplanted to the arterial environment. From the beginning of the development we tried to keep the total volume of the circuit relatively small. At present it comprises approximately $20 \mathrm{ml}$ which is substantially lower compared to other systems which use volumes up to $500 \mathrm{ml}[30,34]$. If necessary the circuit can be scaled down even further to a volume of approximately $10 \mathrm{ml}$. Exogenous substances can be added in a defined concentration with a reasonable and affordable consumption of material, even during long-term experiments with repeated changes of medium and substances. One conceivable scenario is the induction of an inflammatory reaction in the vein followed by the addition of recently developed anti-inflammatory drugs [35,36]. Another most obvious application is the use of small molecules which have shown their anti-angiogenic potential in vitro [37]. Our system might unveil novel aspects about the activity of such molecules as the affected endothelial cells are located in their natural environment and maintain their physiological interactions with other cell types.

\section{Conclusions}

In summary, we have developed a novel ex vivo perfusion system which maintains human veins viable for up to two weeks under a low pressure profile. The setup guarantees a tightly controlled and stable perfusion rate and the system proved to be suitable to record alterations in gene and protein expression induced by different perfusion profiles. Further advantages of our system are a total flexibility concerning the size of 
potential vessels and almost infinite possibilities in various research areas by the addition of defined amounts of exogenous substances into the circuit. Our ex vivo perfusion system and its applications may, therefore, help to improve the long-term patency of human bypass grafts.

\section{Additional material}

Additional File 1: Figure S1. Histological analysis of a representative formalin fixed and paraffin-embedded HSVG after perfusion with different pressure profiles and hematoxylin/eosin staining. The control represents the unperfused vessel. The other parts of the vein were perfused with physiological venous (10 $\mathrm{mmHg}$ ) or arterial pressure $(100 \mathrm{mmHg})$ for the time indicated.

\section{Acknowledgements}

We gratefully acknowledge the excellent support of Christian Becker in the establishment of the controlling software. This work was supported by institutional and departmental sources from the Department of Cardiovascular Surgery of the German Heart Center. The authors gratefully acknowledge the support of the TUM's Faculty Graduate Center Weihenstephan at Technische Universität München.

\section{Author details}

${ }^{1}$ German Heart Center Munich at the Technische Universität München, Department of Cardiovascular Surgery, Lazarettstrasse 36, D-80636 Munich, Germany. ${ }^{2}$ Technische Universität München, Research Center for Nutrition and Food Science, Nutritional Medicine Unit, Gregor-Mendel-Strasse 2, 85350 Freising-Weihenstephan, Germany.

\section{Authors' contributions}

- SD prepared the human saphenous vein grafts and mounted them into the perfusion system, tested the viability of the vein grafts, performed quantitative RT-PCR-analysis and wrote the major part of the manuscript

- SE constructed the set-up of the perfusion system and helped with the development of the software

- CT helped to prepare and mount the HSVGs, participated in the quantitative RT-PCR-analysis and performed the zymographic studies

- US participated in the design and helped to build the perfusion device

- BV harvested the HSVGs for the perfusion system and viability experiments during CABG surgery and helped to draft the manuscript

- MAD participated in harvesting the HSVGs for the perfusion system and viability experiments during CABG surgery and helped to draft the manuscript

- $\mathbf{H H}$ participated in the design and coordination of the study and helped to draft the manuscript

- HL participated in the design of the study, performed statistical analyses and participated in writing of the manuscript

- RL harvested the HSVGs for the perfusion system, participated in the design of the study and its coordination and helped to draft and improve the manuscript

- MK initiated and designed major parts of the study, participated in coordination and writing of the manuscript

All authors read and approved the final manuscript.

\section{Competing interests}

The authors declare that they have no competing interests.

Received: 18 May 2011 Accepted: 21 July 2011 Published: 21 July 2011

\section{References}

1. Motwani JG, Topol EJ: Aortocoronary saphenous vein graft disease: pathogenesis, predisposition, and prevention. Circulation 1998, 97:916-931.

2. Campeau L, Enjalbert M, Lespérance J, Bourassa MG, Kwiterovich P Jr, Wacholder S, Sniderman A: The relation of risk factors to the development of atherosclerosis in saphenous-vein bypass grafts and the progression of disease in the native circulation. A study 10 years after aortocoronary bypass surgery. New Engl J Med 1984, 311:1329-1332.

3. Dashwood MR: Endothelin-1 and vein graft occlusion in patients undergoing bypass surgery. Eur J Clin Invest 2009, 39:78-87.

4. Fitzgibbon GM, Kafka HP, Leach AJ, Keon WJ, Hooper GD, Burton JR: Coronary bypass graft fate and patient outcome: angiographic follow-up of 5,065 grafts related to survival and reoperation in 1,388 patients during 25 years. $J \mathrm{Am}$ Coll Cardiol 1996, 28:616-626.

5. Grondin CM, Campeau L, Lespérance J, Enjalbert M, Bourassa MG: Comparison of late changes in internal mammary artery and saphenous vein grafts in two consecutive series of patients 10 years after operation. Circulation 1984, 70:1208-1212.

6. Kloppenburg GT, Grauls GE, Bruggeman CA, Stassen FR: Adenoviral activin A expression prevents vein graft intimal hyperplasia in a rat model. Interact Cardiovasc Thorac Surg 2009, 8:31-34. 
7. Gusic RJ, Myung R, Petko M, Gaynor JW, Gooch KJ: Shear stress and pressure modulate saphenous vein remodeling ex vivo. J Biochmech 2005, 38:1760-1769.

8. Thomas AC, Newby AC: Effect of Matrix Metalloproteinase-9 knockout on vein graft remodelling in mice. J Vasc Res 2010, 47:299-308

9. Malek AM, Alper SL, Izumo S: Hemodynamic shear stress and its role in atherosclerosis. JAMA 1999, 282:2035-2042.

10. Smith $\mathrm{S}$, Austin $\mathrm{S}$, Wesson GD, Moore CA: Calculation of wall shear stress in left coronary artery bifurcation for pulsatile flow using two-dimensional computational fluid dynamics. Conf Proc IEEE Eng Med Biol Soc 2006, 1:871-874.

11. Muto A, Model L, Ziegler K, Eghbalieh SD, Dardik A: Mechanisms of vein graft adaptation to the arterial circulation: insights into the neointimal algorithm and management strategies. Circ J 2010, 74:1501-1512.

12. Doronzo G, Russo I, Mattiello L, Trovati M, Anfossi G: Homocysteine rapidly increases matrix metalloproteinase-2 expression and activity in cultured human vascular smooth muscle cells. Role of phosphatidyl inositol 3-kinase and mitogen activated protein kinase pathways. Thromb Haemost 2005, 94:1285-1293.

13. Newby AC: Dual role of matrix metalloproteinases (matrixins) in intimal thickening and atherosclerotic plaque rupture. Physiol Rev 2005, 85:1-31.

14. Whatling C, MCPheat W, Hurt-Camejo E: Matrix management: assigning different roles for MMP-2 and MMP-9 in vascular remodelling. Arterioscl Thromb Vasc Biol 2004, 24:10-11.

15. Wilson YG, Davies AH, Southgate K, Currie IC, Sheffield E, Baird RN, Lamont PM, Angelini GD: Vein quality influences neointimal hyperplasia in an organ culture model of human saphenous vein. Eur J Vasc Endovasc Surg 1997, 13(6):557-562.

16. Berceli SA, Jiang Z, Klingman NV, Pfahnl CL, Abouhamze ZS, Frase CD, Schultz GS, Ozaki CK: Differential expression and activity of matrix metalloproteinases during flow-modulated vein graft remodeling. J Vasc Surg 2004, 39(5):1084-1090.

17. Patterson MA, Leville CD, Hower CD, Jean-Claude JM, Seabrook GR, Towne JB, Cambria RA: Shear force regulates matrix metalloproteinase activity in human saphenous vein organ culture. J Surg Res 2001, 95(1):67-72.

18. Bernas T, Dobrucki J: Mitochondrial and Nonmitochondrial Reduction of MTT: Interaction of MTT with TMRE, JC-1, and NAO Mitochondrial Fluorescent Probes. Cytometry 2002, 47:236-242.

19. Burunova W, Gisina AM, Kholodenko IV, Lupatov AY, Shragina OA, Yarygin KN: Standardization of Biochemical Profile of Mesenchymal Cell Materials by Probing the Level of Dehydrogenase Activity. Bull Exp Biol Med 2010, 149(4):497-501.

20. Castro MM, Rizzi E, Figueiredo-Lopes L, Fernandes K, Bendhack LM, Pitol DL, Gerlach RF, Tanus-Santos JE: Metalloproteinase inhibition ameliorates hypertension and prevents vascular dysfunction and remodeling in renovascular hypertensive rats. Atherosclerosis 2008, 198:320-331.

21. Derosa G, D'Angelo A, Ciccarelli L, Piccinni MN, Pricolo F, Salvadeo S, Montagna L, Gravina A, Ferrari I, Galli S, Paniga S, Tinelli C, Cicero AF: Matrix metalloproteinase-2, -9 , and tissue inhibitor of metalloproteinase-1 in patients with hypertension. Endothelium 2004, 13:227-231

22. Mavromatis K, Fukai T, Tate M, Chesler N, Ku DN, Galis ZS: Early effects of arterial hemodynamic conditions on human veins perfused ex vivo. Arterioscler Thromb Vasc Biol 2000, 20:1889-1895.

23. Tran-Son-Tay R, Hwang M, Garbey M, Jiang Z, Ozaki CK, Berceli SA: An experiment-based model of vein graft remodeling induced by shear stress. Ann Biomed Eng 2008, 36(7):1083-1091, Epub 2008 Apr 15.

24. Hilker M, Längin T, Hake U, Schmid FX, Kuroczynski W, Lehr HA, Oelert H, Buerke M: Gene expression profiling of human stenotic aorto-coronary bypass grafts by cDNA array analysis. Eur J Cardiothorac Surg 2003, 23:620-625.

25. Marques FZ, Campain AE, Yang YH, Morris BJ: Meta-analysis of genome-wide gene expression differences in onset and maintenance phases of genetic hypertension. Hypertension 2010, 56:319-324.

26. Puig O, Wang IM, Cheng P, Zhou P, Roy S, Cully D, Peters M, Benita Y, Thompson J, Cai TQ: Transcriptome profiling and network analysis of genetically hypertensive mice identifies potential pharmacological targets of hypertension. Physiol Genomics 2010, 42:24-32.

27. Jiang Z, Tao M, Omalley KA, Wang D, Ozaki CK, Berceli SA: Established neointimal hyperplasia in vein grafts expands via TGF-beta-mediated progressive fibrosis. Am J Physiol Heart Circ Physiol 2009, 297(4):H1200-1207, Epub 2009 Jul 17.

28. Miyakawa AA, Dallan LAO, Lacchini S, Borin TF, Krieger JE: Human saphenous vein organ culture under controlled hemodynamic conditions. Clinics 2008, 63:683-688.

29. Saucy F, Probst H, Alonso F, Bérard X, Déglise S, Dunoyer-Geindre S, Mazzolai L, Kruithof E, Haefliger JA, Corpataux JM: Ex vivo pulsatile perfusion of human spahenous veins induces intimal hyperplasia and increased levels of the plasminogen activator inhibitor 1. Eur Surg Res 2010, 45:50-59.

30. Porter KE, Nydahl S, Dunlop P, Varty K, Thrush AJ, London NJM: The development of an in vitro flow model of human saphenous vein graft intimal hyperplasia. Cardiovasc Res 1996, 31:607-614.

31. Paroz A, Probst H, Saucy F, Mazzolai L, Rizzo E, Ris HB, Corpataux JM: Comparison of morphological and functional alterations of human saphenous veins after seven and fourteen days of ex vivo perfusion. Eur Surg Res 2004, 36:274-281.

32. Park H, Berzin I, De Luis J, Vunjak-Novakovic G: Evaluation of silicone tubing toxicity using tobacco BY2 culture. In Vitro Cell Dev Biol -Plant 2005, 41(4):555-560.

33. Rey J, Probst H, Mazzolai L, Bosman FT, Pusztaszeri M, Stergiopulos N, Ris HB, Hayoz D, Saucy F, Corpataux JM: Comparative assessment of intimal hyperplasia development after 14 days in two different experimental settings: tissue culture versus ex vivo continuous perfusion of human saphenous vein. J Surg Res 2004, 121:42-49.

34. Labadie RF, Antaki JF, Williams JL, Katyal S, Ligush J, Watkins SC, Pham SM, Borovetz HS: Pulsatile perfusion system for ex vivo investigation of biochemical pathways in intact vascular tissue. Am J Physiol 1996, 270:H760-768.

35. Law MR, Morris JK, Wald NJ: Use of blood pressure lowering drugs in the prevention of cardiovascular disease: meta-analysis of 147 randomised trials in the context of expectations from prospective epidemiological studies. BMJ 2009, 338:b1665.

36. Roth AG, Drescher D, Yang Y, Redmer S, Uhlig S, Arenz C: Potent and selective inhibition of acid sphingomyelinase by biphosphonates. Angew Chemie Int Ed Engl 2009, 48:7560-7563. 
37. Zahler S, Liebl J, Fürst R, Vollmar AM: Anti-angiogenic potential of small molecular inhibitors of cyclin dependent kinases in vitro. Angiogenesis 2010, 13:239-249

doi:10.1186/1475-925X-10-62

Cite this article as: Dummler et al:: Pulsatile ex vivo perfusion of human saphenous vein grafts under controlled pressure conditions increases MMP-2 expression. BioMedical Engineering OnLine 2011 10:62.

Submit your next manuscript to BioMed Central and take full advantage of:

- Convenient online submission

- Thorough peer review

- No space constraints or color figure charges

- Immediate publication on acceptance

- Inclusion in PubMed, CAS, Scopus and Google Scholar

- Research which is freely available for redistribution 